\title{
Universal triangular spectra in parametrically-driven systems
}

\author{
Nail Akhmediev a ${ }^{\text {Adrian Ankiewicz }}{ }^{\mathrm{a}, *}$, J.M. Soto-Crespo $^{\mathrm{b}}$, John M. Dudley ${ }^{\mathrm{c}}$ \\ a Optical Sciences Group, Research School of Physics and Engineering, Institute of Advanced Studies, The Australian National University, Canberra ACT 0200, Australia \\ ${ }^{\mathrm{b}}$ Instituto de Óptica, C.S.I.C., Serrano 121, 28006 Madrid, Spain \\ ' Institut FEMTO-ST, UMR 6174 CNRS-Université de Franche-Comté, 25030 Besançon, France
}

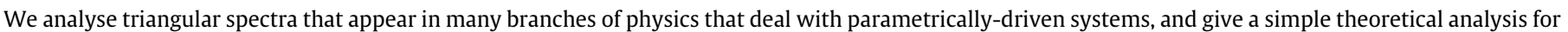

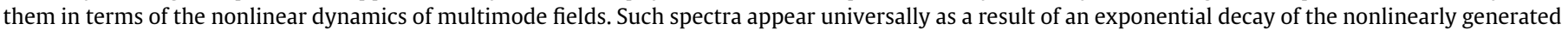

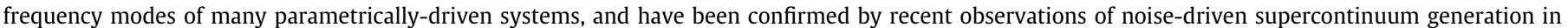

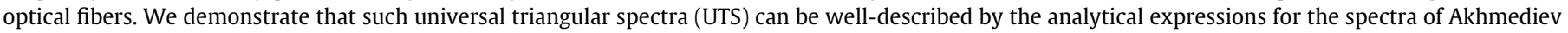
breather $(\mathrm{AB})$ solutions at the point of maximal compression.

\section{Introduction}

A defining feature of parametric systems in physics is the generation of multiple frequency components as a result of nonlinear energy transfer from an initial single-mode spectral excitation. In many systems, the spectra of the evolving field are associated with an exponentially decaying energy transfer from the pump frequency, leading to a characteristic universal triangular spectral (UTS) form when, as is usual, the energy content is analyzed or measured logarithmically.

Examples of such spectra abound in physics, and include spin waves in ferrites (yttrium iron garnet) (see Fig. 3 of [1]) and parametrically-generated second sound waves in superfluid $\mathrm{He}^{4}$ (see Fig. 3 of [2]). On the other hand, the explanations that have been given for the specific spectral broadening in these works are complicated and not completely satisfactory. A more recent result comes from the physics of capillary waves [3], where results have been obtained through careful experiments and are presented in a significantly clearer way. This has allowed the authors to make a specific link between the parametrically-driven system and the nonlinear dynamics of modulation instability and rogue waves that leads to the appearance of triangular spectra associated with capillary waves on the excited surface of water.

The most recent example of the excitation of triangular spectra is from optics. In fact, the initial stage of supercontinuum generation in optical fibers, starting with a continuous wave, has this specific triangular spectrum, with increasing width $[4,5]$. Until recently, this phenomenon has been explained using ideas of

\footnotetext{
* Corresponding author.

E-mail address: ana124@rsphysse.anu.edu.au (A. Ankiewicz).
}

multiple four-wave mixing [4] or soliton fission [6], but a simpler and more general explanation, based on modulation instability and parametric frequency conversion, can also be given for this case.

Clearly, parametric processes are the major cause of the transfer of energy between spectral components. In most cases (including surface waves [3] and optical fibres [7]), four-wave mixing (FWM) [8] processes play the major role, and there is no doubt that a set of coupled equations relating a multiplicity of frequency components can describe the above phenomena [4]. However, when proceeding in such a manner, the analysis can become cumbersome and difficult to understand in clear physical terms. Fortunately, there is a way to reduce these complications by using a single (master) equation that intrinsically describes all the frequency transformations and their mutual interactions. For fourwave mixing, this "master equation" is the well-known nonlinear Schrödinger equation (NLSE).

For the NLSE, it is possible to show that the self-phase modulation term adequately describes the interaction between a potentially infinite number of frequency components. This would be extremely difficult to treat analytically via a coupled mode formalism. While, in some cases, a truncated mode approach, considering only a limited number of components, can be used [9,10], it has recently been appreciated that there exist (and have indeed existed in the literature for some years) a number of analytic techniques that allow the NLSE to be solved more easily, allowing a more complete description of the underlying physics than that provided by truncated models.

This is particularly the case for the phenomenon of modulation instability (MI), where a single spectral component in an NLSE system, together with noise or low amplitude sidebands, undergoes "instability" during propagation and the energy "drifts" from the central frequency component to the sidebands. MI and four- 

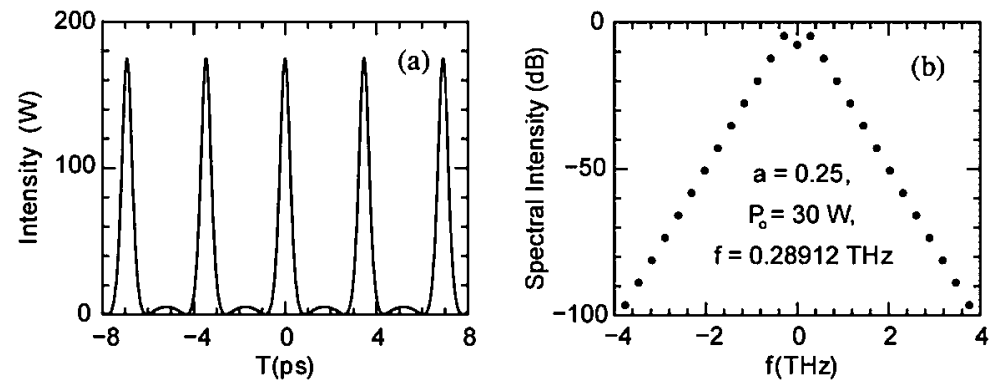

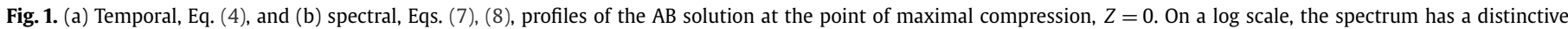
triangular shape.

wave mixing are, in a sense, different views of the same process, in which there is a dynamic energy transfer between adjacent sidebands. A defining feature of the many previous numerical studies of the process has been the observation of a (logarithmic) triangular spectrum. Although MI and four wave mixing processes within the NLSE are often studied via numerical methods, the underlying dynamics can, in fact, be described by a series of exact solutions of the NLSE, now often referred to as Akhmediev breathers (AB) [11-15]. Significantly, the spectra of the AB solutions are logarithmically triangular, and, in optics they reflect the evolution dynamics seen in experiments, possessing the tendency to increase in width from a single frequency to a wide spectrum covering almost the whole optical range [7].

In the present work, we concentrate on the evolution of spectra that can be described by the exact and simple analytical expressions described by the $A B$ theory. Although we focus on particular systems of optics and hydrodynamics, our analysis should be well-suited to describing results in other fields [1-4] and should motivate further studies in systems such as cold atoms and condensates where NLSE-like dynamics are also observed.

\section{Modulation instability theory}

The MI theory from the 1986 paper [17] predicts general results for the distance-dependent evolution of the amplitudes of the $A B$ spectral sidebands. The results here show this spectral evolution more clearly than in Ref. [7], and confirm the theoretical predictions explicitly. We start the analysis with the NLSE in dimensional form [18]

$i \frac{\partial A}{\partial Z}+\frac{\beta_{2}}{2} \frac{\partial^{2} A}{\partial T^{2}}+\gamma|A|^{2} A=0$,

where the coefficients are defined in [18]. Using a simple change of variables,

$Z=z L_{N L}, \quad T=t \sqrt{\left|\beta_{2}\right| L_{N L}}, \quad A=u \sqrt{P_{0}}$,

where $L_{N L}=\left(\gamma P_{0}\right)^{-1}$, Eq. (1) can be converted to its dimensionless form:

$i u_{z}+\frac{1}{2} u_{t t}+|u|^{2} u=0$.

Thus, any solution of (3) can be easily transformed into a solution of (1), and vice versa. The parameter $P_{0}$ in the transformation is the peak power if we are dealing with pulses and is the average power if we deal with continuous waves.

Eq. (1) has a solution in the form of the $A B$ given by:

$A(Z, T)$

$$
=\sqrt{P_{0}} \exp \left(i \frac{Z}{L_{N L}}\right)
$$

$$
\times \frac{(1-4 a) \cosh \left(\frac{b Z}{L_{N L}}\right)+i b \sinh \left(\frac{b Z}{L_{N L}}\right)+\sqrt{2 a} \cos \left(\omega_{\bmod } T\right)}{\sqrt{2 a} \cos \left(\omega_{\bmod } T\right)-\cosh \left(\frac{b Z}{L_{N L}}\right)} .
$$

This solution shows growth-return evolution over $-\infty<Z<\infty$ and represents a family of solutions with a variable independent parameter $\omega_{\text {mod }}$, corresponding to a single frequency modulation on the initial continuous wave (CW) solution. The coefficients $a$ and $b$ depend on $\omega_{\bmod }$ and are defined by:

$a=\frac{1}{2}\left[1-\left(\frac{\omega_{\text {mod }}}{\omega_{c}}\right)^{2}\right]$

$b=\sqrt{8 a(1-2 a)}$

where

$\omega_{c}^{2}=4 \gamma P_{0} /\left|\beta_{2}\right|$

and $P_{0}$ the power of the $\mathrm{CW}$ field at large $|Z|$. The solution is valid over the range of modulation frequencies that experience $\mathrm{MI}$ gain, namely $0<\omega_{\text {mod }}<\omega_{c}$. The coefficient $a$ then varies in the interval $0<a<1 / 2$, while the parameter $b(>0)$ governs the MI growth. The maximum gain condition, $b=1$, occurs for $a=1 / 4$, i.e. $\omega_{\bmod }=\omega_{c} / \sqrt{2}$.

Initially, at $z \rightarrow-\infty$, the solution is a continuous wave with a constant amplitude which subsequently develops a small modulation at $\omega_{\text {mod }}$ when $z$ becomes finite. The modulation increases when $z \rightarrow 0$ and has maximal compression of the pulses in a train when $Z=0$. The amplitude profile of the solution at $Z=0$ for maximal gain is shown in Fig. 1(a).

In the frequency domain, the $A B$ spectrum consists of discrete frequency sideband modes with separation $\omega_{\text {mod }}$, and amplitudes that evolve in $z$ according to:

$$
\begin{aligned}
f_{0}(z)= & e^{i z} \times \frac{i \sqrt{2} \sinh (b z)+\sqrt{2} \cosh (b z)-\sqrt{\cosh (2 b z)}}{\sqrt{\cosh (2 b z)}}, \\
f_{n}(z)= & e^{i z} \frac{i \sqrt{2} \sinh (b z)+\sqrt{2} \cosh (b z)}{\sqrt{\cosh (2 b z)}} \\
& \times(\sqrt{2} \cosh (b z)-\sqrt{\cosh (2 b z)})^{|n|} .
\end{aligned}
$$

Here $f_{0}(z)$ describes the pump amplitude and the $f_{n}(z)$, where $n= \pm 1, \pm 2, \pm 3, \ldots$, are the sideband amplitudes. We have used the relation $\cosh (2 b z)=2 \cosh ^{2}(b z)-1$ to simplify the formulae given in [17]. Using Eq. (2), the spectrum can be converted to dimensional units.

A unique feature of the spectrum is that, for $|n|>0$,

$\log \left(\frac{f_{n}(0)}{\sqrt{2}}\right)=-|n| \log (1+\sqrt{2}) \approx-0.88|n|$.

Hence, the points plotted in Fig. 1(b) $(|n|>0)$ form two straight lines. At $z \rightarrow-\infty$, the spectrum consists of a single central nonzero component that contains all the energy. At finite $z$, the 
nearest sidebands appear at the expense of the central component. The sidebands grow and create additional sidebands, thus filling the whole spectrum with equidistant components. The spectrum given by Eqs. (7), (8) at the point $Z=0$ is shown in Fig. 1(b). It is clearly triangular and represents the result of successive energy transfer between the spectral components from the central mode to sidebands due to the four-wave mixing process. On further evolution, when $z>0$, the tendency is reversed and energy flows from the sidebands back to the central mode.

Initially, only the pair of sidebands which is closest to the central component is unstable due to MI (see Fig. 2(a)). As soon as this pair grows, FWM coupling transfers energy to higher frequencies, generating additional sidebands even at frequencies where amplitudes initially were zero. Describing these dynamics in terms of a set of coupled equations for this potentially infinite number of spectral components is difficult [4], but in fact the recent realisation that this process is described by the $A B$ theory means that such a multi-mode description is in fact not necessary: the exact $A B$ solution intrinsically and exactly describes the ensemble of FWM processes that occur in this evolution.

$A B$ solutions are heteroclinic orbits (see Fig. 1 of [17]) that start from a CW which is a saddle point of the dynamical system. Thus, a single growth-return evolution will be observed only if the trajectory starts along this heteroclinic one. If we choose a starting position at another nearby point, we generally will obtain periodic motion (see Eq. (18) of [17]). For example, the dynamics around the heteroclinic $A B$ solutions can be studied by numerically solv-
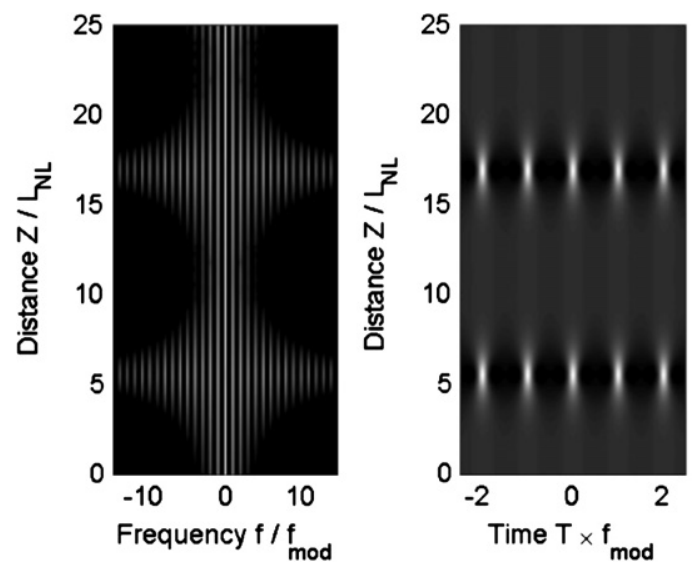

Fig. 2. Spectral and temporal profiles of the $A B$ solution over two periods of evolution. ing the NLSE for the evolution of a modulated CW input field of the form

$$
A\left(Z=Z_{0}, T\right)=\sqrt{P_{0}}\left[1+\alpha_{\text {mod }} \cos \left(\omega_{\text {mod }} T\right)\right] .
$$

In this case, we obtain the periodic motion shown in Fig. 2. The period along the $z$-direction in this motion depends on how much the initial condition deviates from the heteroclinic orbit, i.e. it depends on the value of $\alpha_{\text {mod }}$. In order to obtain an infinite period, $\alpha_{\text {mod }}$ has to be a complex number.

For waves in an optical fiber, we can estimate the real values of the magnitudes involved. We use the following values of the fiber parameters at $1550 \mathrm{~nm}: \beta_{2}=20 \mathrm{ps}^{2} \mathrm{~km}^{-1}, \gamma=1.1 \mathrm{~W}^{-1} \mathrm{~km}^{-1}$, and $P_{0}=30 \mathrm{~W}$. Then MI gain is observed for modulation frequencies $f_{m}=\omega_{\bmod } / 2 \pi$ in the range $\left[0, f_{c}\right]$ where $f_{c}=\omega_{c} / 2 \pi=$ $408.88 \mathrm{GHz}$. The maximum gain at $a=0.25$ corresponds to a modulation frequency $f_{m}=f_{c} / \sqrt{2}=289.12 \mathrm{GHz}$. The spectral and temporal evolution for this case with $\alpha_{\text {mod }}=0.01$ are shown in gray-scale in Fig. 2. We show the temporal intensity $|A(Z, T)|^{2}$ and spectral intensity $|\tilde{A}(Z, f)|^{2}$, where $A(Z, T) \leftrightarrow \tilde{A}(Z, f)$ are a Fourier transform pair. The propagation distance $z$ is scaled relative to the characteristic nonlinear length $L_{N L}=\left(\gamma P_{0}\right)^{-1}=$ $30.3 \mathrm{~m}$.

An alternative and visually more attractive way to view the spectral evolution is as a surface plot. This is presented in Fig. 3. Two growth-return cycles of spectral evolution are clearly seen. The central component $f_{0}$ is restored in between the cycles (point B). The initial spectrum is completely restored, i.e. all higher-order components disappear and the spectrum consists of only the central component and the pair of nearest sidebands.

To compare with the $A B$ theory for the spectral amplitudes, we follow the evolution along the "return phase" of the trajectory from the first point of spectral expansion where there is maximum conversion into the sidebands to the next point in the evolution where the energy is transferred back to the pump. These two points are identified as A and B, respectively, in Fig. 3. Point $A$ is the location of the maximally-compressed $A B$ pulse that we can associate with $z=0$ in Eqs. (7) and (8). From the numerical simulations, we can readily determine the intensity of each sideband and then compare with the predictions of Eqs. (7) and (8).

The results are shown in Fig. 4. Here the origin, $z=0$, corresponds to point $A$ above, the markers represent the numericallydetermined sideband amplitudes and the solid lines are the predictions of Eqs. (7) and (8). There is excellent agreement.

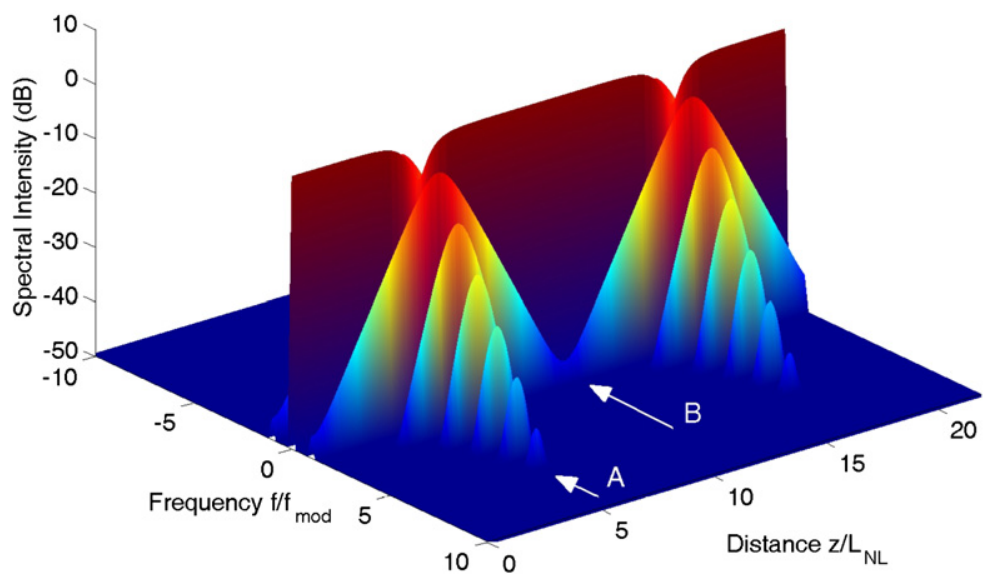

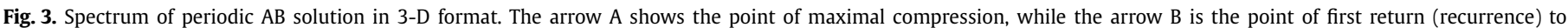
the starting conditions. 


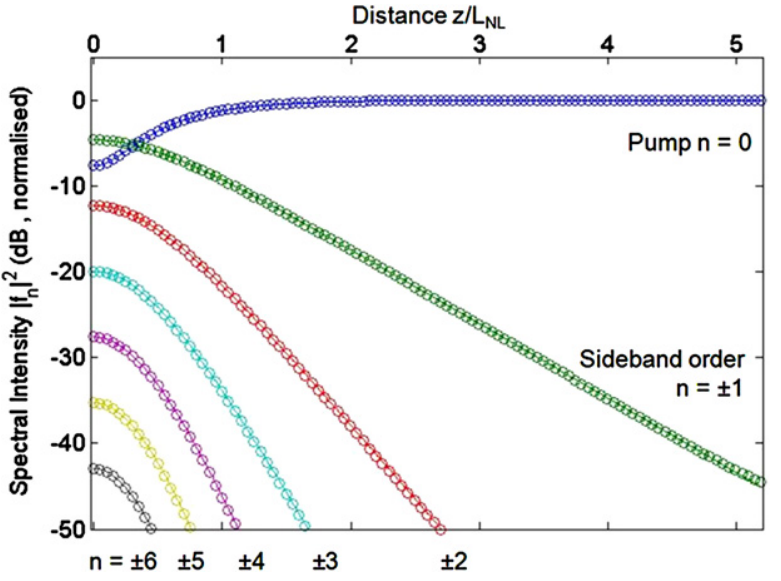

Fig. 4. Comparison of spectral components, $f_{n}$, for the pump $n=0$ and sidebands $n= \pm 1 \ldots \pm 6$ obtained numerically (dots), and from exact solutions (7) and (8). The range of $z$ here is chosen between the points A and B in Fig. 3 .

More accurate spectra for a higher-valued $\alpha_{\bmod }$ should be based on exact solution in terms of elliptic Jacobi functions given in the paper [17] (see Eq. (18) therein when $\kappa \rightarrow 1$ ). These may have direct application in analytic approaches to understanding beat signal reshaping and frequency comb generation.

\section{Multi-frequency initial modulation}

When the initial modulation consists of only a single pair of sidebands, the analysis is reasonably simple, as it was shown in the previous section. The case of two or more sidebands can also be analyzed using the nonlinear superposition of $A B$ solutions. There is a well-developed technique for constructing higher-order solutions that start with modulation instability $[16,19]$. The results are cumbersome and will not be reproduced here. Another difficulty is calculating Fourier spectra for higher-order solutions as there is no technique for deriving analytic spectra in those cases.

Despite the absence of analytical solutions, we can still use the fact that the system described by the NLSE is integrable to present a qualitative analysis for triangular spectra in this case. Indeed, each $\mathrm{AB}$ component in the nonlinear superposition defined by the NLSE is independent of the others, in the sense that it can be used to construct a nonlinear superposition. Then, for every additional pair of sidebands, the spectra are triangular and evolve periodically, just as in Fig. 3. An important point here is that we do not have to deal with the frequency that provides us with the highest growth rate. As the growth rate depends on the frequency within the instability band, each component grows at its own rate. Their corresponding points A for the components with small growth rate may move infinitely far from the beginning. The periods of evolution for different sidebands also vary, depending on the amplitude of each component. Thus, for two or more sidebands, at every $z$ there will be a triangular spectrum related to one of the components. Thus, dealing with nonlinear superposition, we cannot expect a complete recurrence to the original mode. Moreover, if the initial modulation is chaotic, i.e. it contains all frequency components within the gain bandwidth, there will be a continuous spectrum at any $z$.

The qualitative behaviour described above is confirmed using numerical simulations starting from a CW with multiple frequency modulation. One example is shown in Fig. 5. Ten initial small spectral components within the instability band used for this simula-

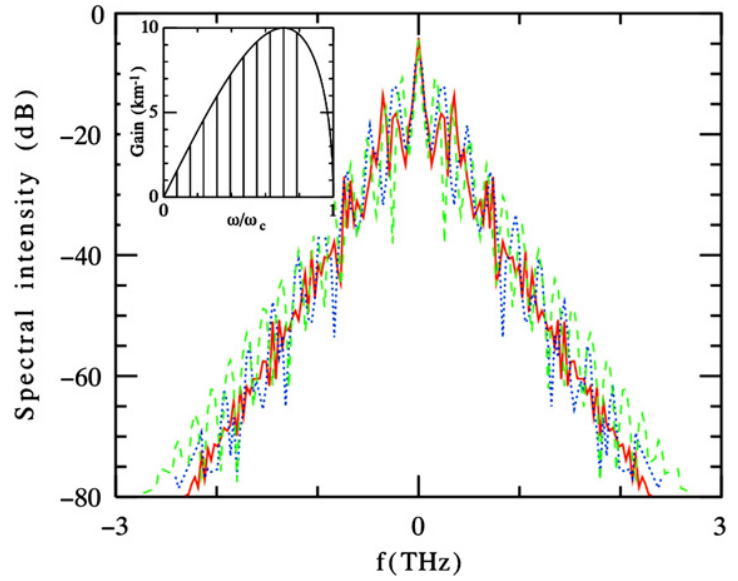

Fig. 5. (Color online.) The spectra of the field with initial modulation given by Eq. (10) at distances of propagation $Z=10 L_{N L}$ (red solid line), $20 L_{N L}$ (blue dotted line), and $30 L_{N L}$ (green dashed line). Inset: MI gain curve with vertical bars that show the spectral components within the gain curve relating to the initial conditions (10).

tion are shown in the inset of Fig. 5. Specifically, we choose the initial condition in the form:

$A(0, T)=\sqrt{30}\left[1+0.001 \sum_{k=1}^{10} \cos \left(\pi k T w_{c} / 40\right)\right]$.

Its spectrum consists of the central component containing almost all energy and ten small equal sidebands at each side. Each sideband pair grows according to its particular growth rate and, after the initial stage, the spectrum assumes a steady triangular shape. If we ignore small deviations, the spectrum hardly changes during further propagation. The different curves shown in Fig. 5 for various distances of propagation demonstrate the convergence to a steady triangular spectrum.

Convergence takes place for random initial modulation as well. Suppose, we take all spectral components with small average amplitudes, namely we choose as the initial condition:

$A(0, T)=\sqrt{30}[1+0.0001 a(T)]$,

where $a(T)$ is a stochastic Gaussian-distributed variable with unit variance and Gaussian correlation function. The resulting spectra for this case, after the initial stage of evolution has passed, are shown in Fig. 6. The function $a(T)$ has been chosen as purely real (red solid curve) or imaginary (blue dashed curve). Although initially the spectra may develop with different speeds, the final result is always the same, as we can see from Fig. 6 . After reaching the "steady" state, around $Z=20 L_{N L}$, the width of the triangular spectrum, on average, does not change.

Comparing the spectra in Fig. 6 with those in Fig. 5 shows that random and regular perturbations lead to triangular spectra with the same width. Moreover, the width coincides with the width of the triangular spectra given by the analytical expressions of Eqs. (7), (8), and shown in Fig. 1. This is a result of independent evolution of a multiplicity of $\mathrm{ABs}$ in the complex field that have their points of maximum compression (B) distributed homogeneously along the $z$-axis. This means that Eqs. (7), (8) provide analytic results for universal triangular spectra in systems governed by the NLSE.

Clearly, any additional term in the NLSE that lifts integrability results in deviations from the triangular spectra. This can be seen in Fig. 3 of [7]. The spectra that develop at the initial stages of MI are triangular. However, the influence of Raman and higher-order 

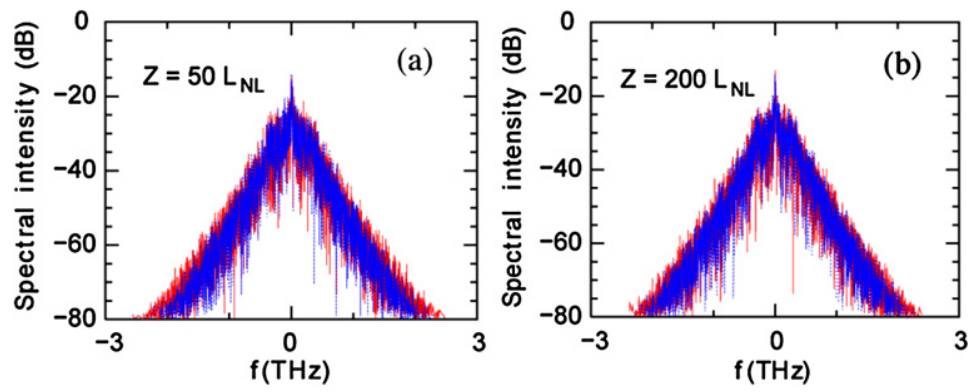

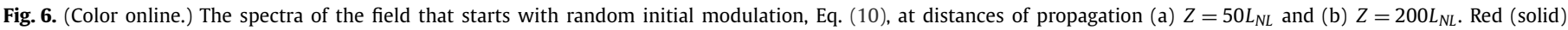
curves are calculated for purely real initial perturbation while blue (dashed) curves are for purely imaginary one.
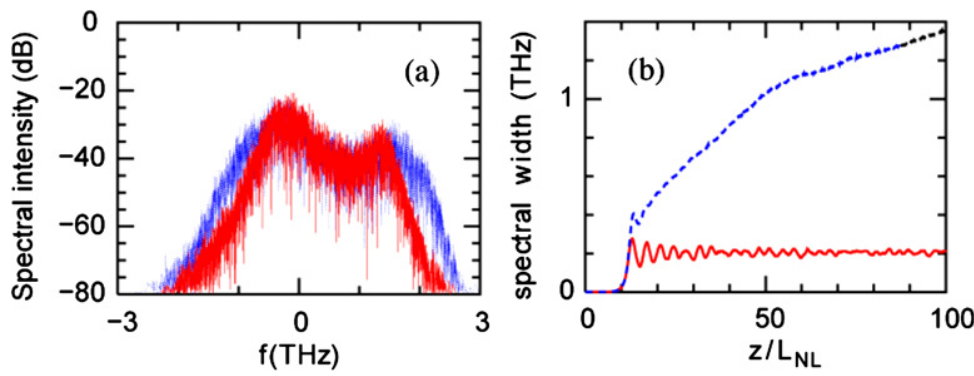

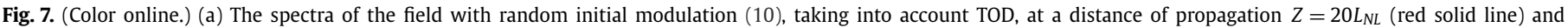
$Z=30 L_{N L}$ (dashed blue line). (b) Evolution of spectral width in $z$ with (blue dashed curve) and without (red solid curve) TOD.

dispersion terms create asymmetry and result in a wide supercontinuum. One more example is shown in Fig. 7(a). Here we use the initial conditions given by Eq. (11), but the NLSE has been modified with an additional third-order dispersion (TOD) term, $\beta_{3} \frac{\partial^{3} A}{\partial T^{3}}$, with a small coefficient, $\beta_{3}=1.557 \mathrm{ps}^{3} / \mathrm{km}$. At small $z$, triangular spectra are generated (not shown). However, by $Z=20 L_{N L}$, the asymmetry has developed, and later on during the propagation $\left(Z=30 L_{N L}\right)$, it creates a wide supercontinuum spectrum, as shown in Fig. 7(a). The widths of the spectral curve with, and without, TOD evolve differently, as can be seen from Fig. 7(b). With the pure NLSE, the width reaches a steady value around $0.2 \mathrm{THz}$, while, with TOD, it increases approximately as $\sqrt{z}$.

\section{Conclusion}

Ubiquitous triangular spectra in parametrically-driven systems described by the NLSE can find a simple explanation in terms of exact $A B$ solutions of the nonlinear Schrödinger equation. In particular, the spectra of the $A B$ solutions that start from noise-driven modulation instability demonstrate the triangular shape with varying width. The maximum spectral width is reached at the point of maximal compression of the AB. Modulation instability that starts from a noisy background, with all the frequencies being present, also develops a triangular spectrum whose width is also approximately the same as for the spectrum of a single $A B$ at its widest part.

It is clear that analytic $A B$ solutions of the NLSE provide an exact description of the cascaded parametric interactions that underlie the dynamics of MI, and their use and interpretation eliminates the necessity of using multiple coupled equations. Certainly for some cases, numerical simulations must be used to obtain exact quantitative predictions, but the qualitative dynamics predicted by the $A B$ theory that yield logarithmic triangular spectra appear universal.

\section{Acknowledgements}

N.A. is grateful to Alexander von Humboldt Foundation, as this work was prepared while he was visiting Germany. The authors N.A. and A.A. acknowledge the support of the Australian Research Council (Discovery Project DP0985394). J.M.S.C. acknowledges support from the Spanish Ministerio de Ciencia e Innovación under contracts FIS2006-03376 and FIS2009-09895. J.M.D. thanks the Institut Universitaire de France and the French Agence Nationale de la Recherche projects MANUREVA ANR-08-SYSC-019 and IMFINI ANR-09-BLAN-0065, for support.

\section{References}

[1] I.V. Krutsenko, V.S. L'vov, G.A. Melkov, JETP 48 (1978) 561.

[2] D. Rinberg, V. Cherepanov, V. Steinberg, Phys. Rev. Lett. 78 (1997) 4383.

[3] M. Shats, H. Punzmann, H. Xia, Phys. Rev. Lett. 104 (2010) 104503.

[4] J.R. Thompson, R. Roy, Phys. Rev. A 43 (1991) 4987.

[5] J.M. Dudley, J.R. Taylor, Supercontinuum Generation in Optical Fibers, Cambridge University Press, 2010.

[6] A.V. Husakou, J. Herrmann, Phys. Rev. Lett. 87 (2001) 203901.

[7] J.M. Dudley, G. Genty, F. Dias, B. Kibler, N. Akhmediev, Optics Express 17 (24) (2009) 21497.

[8] R. Stolen, J. Bjorkholm, IEEE J. Quantum Electron. 18 (7) (1982) 1062.

[9] G. Cappellini, S. Trillo, JOSA B 8 (1991) 824.

[10] S. Trillo, S. Wabnitz, Opt. Lett. 16 (1991) 986.

[11] K.B. Dysthe, K. Trulsen, Physica Scripta T82 (1999) 48

[12] V.V. Voronovich, V.I. Shrira, G. Thomas, J. Fluid Mech. 604 (2008) 263.

[13] I. Ten, H. Tomita, Reports of RIAM Symposium No.17SP1-2, Proceedings of a Symposium Held at Chikushi Campus, Kyushu University, Kasuga, Fukuoka, Japan, March 10-11, 2006.

[14] D. Clamond, M. Francius, J. Grue, C. Kharif, European J. Mech. B Fluids 25 (2006) 536.

[15] V.I. Shrira, V.V. Geogjaev, J. Eng. Math. 67 (2010) 11.

[16] N. Akhmediev, V.M. Eleonskii, N.E. Kulagin, Sov. Phys. JETP 62 (1985) 894.

[17] N. Akhmediev, V.I. Korneev, Theor. Math. Phys. (USSR) 69 (2) (1986) 189, Translation from Russian, Theor. Math. Phys. (USSR) 69 (1986) 1089.

[18] G.P. Agrawal, Nonlinear Fiber Optics, Academic Press, NY, 1989.

[19] N. Akhmediev, V.I. Korneev, N.V. Mitskevich, Zh. Eksp. Teor. Fiz. 94 (1988) 159, Sov. Phys. JETP 67 (1988) 89. 